Review began 09/01/2021 Review ended 09/15/2021 Published 09/21/2021

\section{() Copyright 2021}

Bayram et al. This is an open access article distributed under the terms of the Creative Commons Attribution License CC-BY 4.0. which permits unrestricted use, distribution, and reproduction in any medium, provided the original author and source are credited.

\title{
Elevation of Serum Transaminase Levels Due to Favipiravir Use in the Treatment of COVID-19
}

Mehmet Bayram ${ }^{1}$, Ozgur Yildirim ${ }^{2}$, Raye Sevra Ozmen ${ }^{2}$, Beyza Soylu ${ }^{2}$, Ahmet Said Dundar ${ }^{2}$, Ali Riza Koksal $^{3}$, Murat Akarsu ${ }^{2}$, Abdulbaki Kumbasar ${ }^{4}$, Omur Tabak ${ }^{4}$

1. Gastroenterology and Hepatology, Health Sciences University Kanuni Sultan Süleyman Training and Research Hospital, Istanbul, TUR 2. Department of Internal Medicine, Health Sciences University Kanuni Sultan Süleyman Training and Research Hospital, Istanbul, TUR 3. Gastroenterology and Hepatology, Tulane University School of Medicine, New Orleans, USA 4. Department of Internal Medicine, Health Sciences University Kanuni Sultan Suleyman Training and Research Hospital, Istanbul, TUR

Corresponding author: Mehmet Bayram, drmhbayram@gmail.com

\section{Abstract}

Background and aims: Favipiravir is a ribonucleic acid (RNA)-dependent RNA polymerase (RdRP) inhibitor antiviral agent used in the treatment of coronavirus disease-2019 (COVID-19). In this study, we investigated the changes in serum transaminase levels of patients and the relationship between serum transaminase elevation with mortality in patients who were hospitalized with the diagnosis of COVID-19 and received favipiravir treatment.

Materials and methods: 454 patients who received favipiravir and 113 patients who did not receive favipiravir were evaluated. Serum transaminase levels of the patients were compared at baseline and after five days of treatment, and the relationship between serum transaminase elevation and mortality was investigated.

Results: No significant aspartate aminotransferase (AST) or alanine aminotransferase (ALT) elevation was detected due to favipiravir treatment. AST elevation was found, respectively, as 133 (29.3\%), 32 (28.3\%) $(\mathrm{p}=0.100)$, ALT elevation as $112(24.7 \%), 35(29.3 \%)(\mathrm{p}=0.100)$ in the groups receiving and not receiving favipiravir. High AST level was found as a risk factor for mortality in all patient groups $(\mathrm{p}=0.008)$.

Conclusions: There was no statistically significant elevation in serum transaminase levels due to favipiravir use in patients hospitalized for COVID-19. A high level of AST is a significant risk factor to show mortality and intensive care unit (ICU) admission in patients with COVID-19.

Categories: Internal Medicine, Gastroenterology

Keywords: icu admission, mortality, elevated transaminases, covid-19, favipiravir

\section{Introduction}

Coronavirus is a non-segmented single-stranded ribonucleic acid (RNA) virus, and it has six species that cause disease in humans [1]. Among these, SARS-CoV-2 (severe acute respiratory syndrome coronavirus 2) was detected for the first time in China in December 2019 and subsequently spread to more than 100 million people in more than 200 countries, causing a global pandemic. World Health Organization (WHO) declared an emergency in January 2020 and a pandemic on March 11, 2020, the disease name was determined as coronavirus disease-2019 (COVID-19). COVID-19 is a disease that can progress in a broad spectrum from asymptomatic mild illness to severe lung disease [2,3]. Hepatic injury in COVID-19 may develop due to the direct cytopathic effect of the virus, uncontrolled immune response, hypoxia secondary to pneumonia, and the drugs used in treatment $[4,5]$. In the literature, the rate of elevation in serum transaminase levels of patients hospitalized for COVID-19 was detected to be $37.5 \%$ [6]. Also, elevation in serum transaminase levels in COVID-19 patients has been detected to be associated with increased mortality in the literature $[7,8]$.

Favipiravir is an antiviral agent developed in Japan for the treatment of drug-resistant influenza [9]. RNAdependent RNA polymerase (RdRP) plays an important role in coronavirus replication. Favipiravir shows its effect by inhibiting RdRP [9]. The effect of the agent on SARS-CoV-2 has been demonstrated in vitro [10]. In early clinical studies, it was detected that the drug reduced viral load in patients and provided clinical and radiological improvement $[11,12]$. Diarrhea, neutropenia and elevation of serum transaminase levels are the most common side effects of the drug. In a review including patients using favipiravir in the treatment of influenza and COVID-19, elevations in serum transaminase levels were detected in $2 \%$ of patients after drug use [13]. With the use of favipiravir in COVID-19, an elevation in serum transaminase levels was detected in $8.6 \%$ of the patients [14]. In our study, we investigated the elevation of serum transaminase levels and the clinical significance of this situation in groups of patients who received and did not receive favipiravir treatment while in hospital with the diagnosis of COVID-19. 


\section{Materials And Methods \\ Study design and data collection}

The study was conducted retrospectively using the medical records of inpatients with positive COVID-19 real-time polymerase chain reaction (RT-PCR) test with signs of COVID-19 pneumonia on thorax CT (computed tomography) between April 1, 2020, and December 31, 2020, at Kanuni Sultan Suleyman Training and Research Hospital. In the study, 454 patients who received favipiravir and 113 patients who did not receive favipiravir among the patients who were hospitalized with normal aspartate aminotransferase (AST) and alanine aminotransferase (ALT) levels (baseline) were evaluated. Patients in the moderate COVID-19 clinical category (room air $\mathrm{SpO}_{2}<95 \%$, shortness of breath, respiratory rate $>24$, fatigue, and heart rate $>110$ ) were included in the study [15]. According to the COVID-19 treatment protocol of the Turkish Health Ministry, during hospitalization, the patients were administered favipiravir treatment for five days as 3200 $\mathrm{mg}$ on the first day and $1200 \mathrm{mg}$ after that. In addition, subcutaneous enoxaparin treatment was given to the patients. Complete blood count (CBC), AST, ALT, C-reactive protein (CRP), and albumin levels at the beginning of hospitalization of the patients and control serum transaminase levels after five days of treatment were recorded and analyzed. CBC parameters were determined by a hematology analyzer (Mindray BC-6800Plus, Mindray, Shenzen, China). Biochemical parameters were measured by the Roche Cobas Integra 800 (Roche Diagnostic Limited, Switzerland) device. Intensive care unit (ICU) admission and mortality due to the disease were recorded. Patients with viral hepatitis, autoimmune hepatitis, and other chronic liver diseases and those with high basal transaminase levels, and those who started on favipiravir with another drug that could cause transaminase elevation were excluded from the evaluation. In addition, patients with diseases and comorbidities that may cause an increase in serum transaminase levels were not included in the study. After five days of treatment, patients with AST and ALT levels $>40 \mathrm{U} / \mathrm{L}$ in control tests were considered to have elevated serum transaminase levels. The difference between serum transaminase levels and the development of elevated serum transaminases in the groups that received and did not receive favipiravir was analyzed. Also, the relationship between elevation of serum transaminases with the ICU admission and mortality in all patients was investigated. This study was approved by the Local Ethical Committee of Kanuni Sultan Süleyman Training and Research Hospital (approval number: KAEK /2021.01.18) and was conducted following the Declaration of Helsinki.

\section{Statistical analysis}

All analyses were performed using SPSS version 21.0 for Windows (IBM Inc., Chicago, Illinois, USA). The variables were investigated using visual (histograms) and analytical methods (Kolmogorov-Smirnov/ShapiroWilk tests) to determine whether or not they were normally distributed. The Chi-square test was used for comparisons of categorical variables. Ordinal variables and continuous variables that do not have normal distribution were compared by the Mann-Whitney U test. The Student t-test was used to evaluate differences between the two groups in normally distributed continuous variables. For multivariate analysis, the possible factors identified by univariate analysis or recent studies were further entered into the logistic regression analysis to determine independent predictors of ICU admission and mortality. Variables that did not make a significant contribution were excluded from the model using the backward likelihood ratio stepwise method. Hosmer-Lemeshow goodness-of-fit statistics were used to assess the model. A value of p<0.05 (two-sided) was considered statistically significant. Values are presented using means \pm standard deviations for normally distributed and medians and first and third quartiles in the brackets for the non-normally distributed variables.

\section{Results}

In the study, 454 patients who received favipiravir treatment and 113 patients who did not were included. AST elevation was found, respectively, as 133 (29.3\%), 32 (28.3\%; p=0.100), ALT elevation as 112 (24.7\%), 35 (29.3\%; $\mathrm{p}=0.100)$ in the groups receiving and not receiving favipiravir. In the group that received favipiravir, serum AST in $26(\% 5,7)$ patients and serum ALT in $33(\% 7,2)$ patients elevated more than two times the upper limit. Besides that, in the group that did not receive favipiravir, serum AST in four $(\% 3,5)$ patients and serum ALT in six $(\% 5,3)$ patients elevated more than two times the upper limit. There was no statistically significant difference between the two groups in terms of serum transaminase elevation rates. None of the patients developed severe serum transaminase elevation (more than five times the upper limit of normal) and liver failure. There was no statistically significant difference in mortality and need for ICU between the patient groups who received and did not receive favipiravir ( $\mathrm{p}=0.99, \mathrm{p}=0.92$, respectively; Figure 1). 


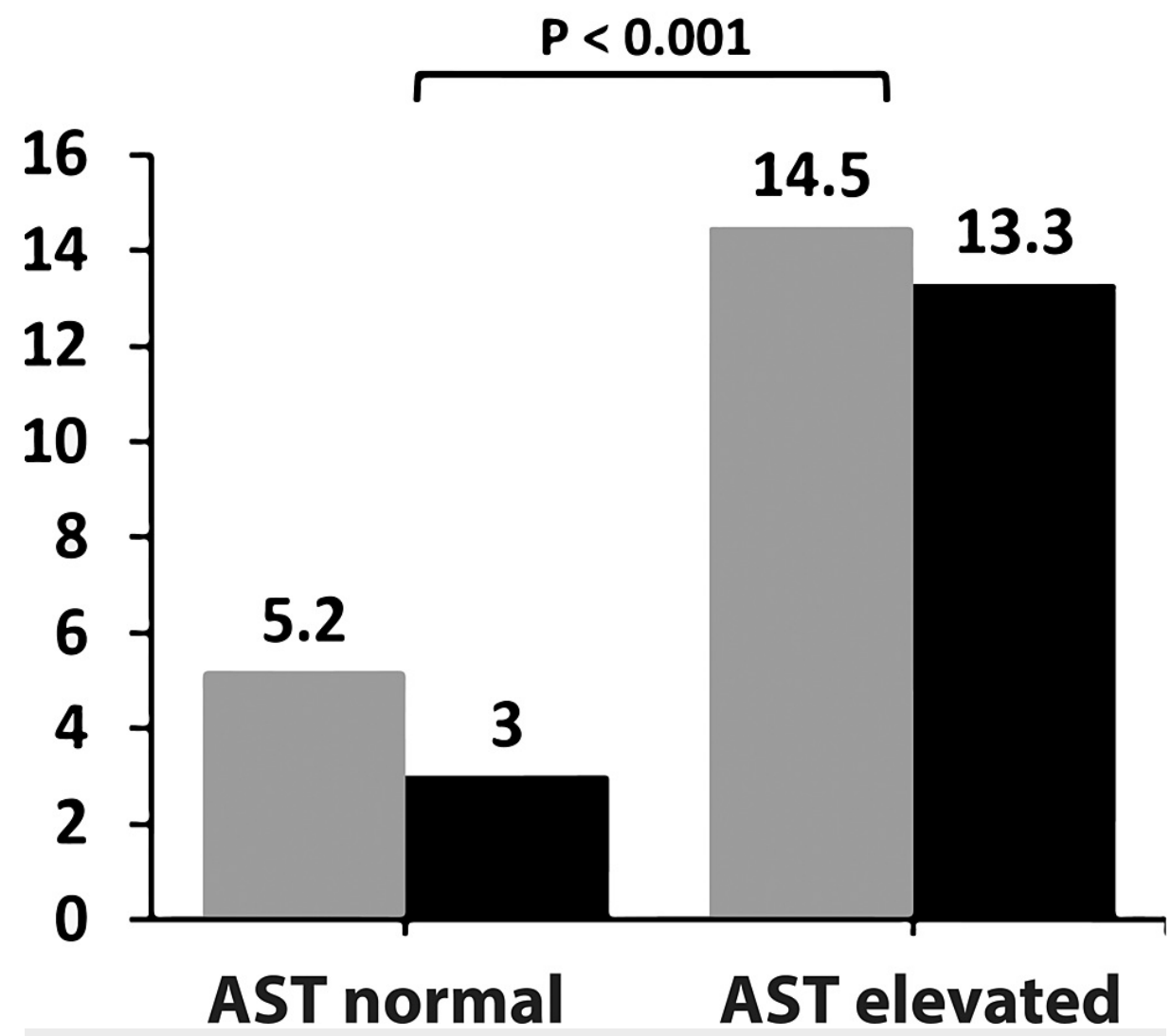

FIGURE 1: Relationship between AST elevation with mortality and ICU admission

Mortality and ICU admission rates were significantly higher in AST elevated group than the AST normal group $(p<$ 0.001). ICU: intensive care unit, AST: aspartate aminotransferase.

Age, gender, hematological and biochemical profile, demographic characteristics, ICU admission, and mortality rates in the patient groups are presented in Table 1. A statistically significant difference was found in the levels of CRP, albumin, and lymphocyte count between the patient groups who received and did not receive favipiravir (Table 1). ICU admission and mortality rates are presented in Table 2 when patients are grouped as AST and ALT elevated and non-elevated. There was a statistically significant difference between AST elevated and AST normal groups in terms of mortality and ICU admission rates ( $<0.001$; Figure 2). There was no statistically significant difference between ALT elevated and ALT normal groups in terms of ICU admission and mortality (Table 2). In univariate and multivariate logistic regression analysis, age, CRP, platelet count, and AST were determined as risk factors for mortality (Table 3). For ICU admission, age, CRP, platelet count, and AST were risk factors in univariate analysis, whereas age, CRP, and platelet count were found as independent risk factors in multivariate analysis (Table 4). 


\section{Cureus}

\begin{tabular}{|c|c|c|c|}
\hline & Patients received favipiravir ( $\mathrm{n}: 454$ ) & Patients who not received favipiravir (n: 113) & P-value \\
\hline Age & $63.0 \pm 15.9$ & $63.8 \pm 11.8$ & 0.590 \\
\hline Male gender $(\mathrm{n} \%)$ & $227(\% 50)$ & $68(\% 58.8)$ & 0.053 \\
\hline AST (baseline) U/L & $26(20-31)$ & $25(21-32)$ & 0.960 \\
\hline ALT (baseline) U/L & $20(16-26)$ & $20(15-30)$ & 0.650 \\
\hline AST (after 5 days of treatment) U/L & $30(21-44)$ & $26(19-48)$ & 0.160 \\
\hline ALI (atter 5 days of treatment) U/L & $24(15-40)$ & $23(16-45)$ & 0.800 \\
\hline Albumin (mg/dL) & $35.1 \pm 4.6$ & $36.8 \pm 5.1$ & 0.003 \\
\hline $\mathrm{CRP}(\mathrm{mg} / \mathrm{dL})$ & $72(26-115)$ & $33(14-81)$ & 0.001 \\
\hline WBC $\left(10^{3} / \mu l\right)$ & $6.6(4.9-9.1)$ & $6.5(5.1-7.8)$ & 0.300 \\
\hline Lymphocyte $\left(10^{3} / \mu \mathrm{l}\right)$ & $1.2(0.8-1.6)$ & $1.4(1-1.8)$ & 0.001 \\
\hline AST elevation ( $\mathrm{n} \%)$ & $133(\% 29.3)$ & $32(\% 28.3)$ & 0.830 \\
\hline ALT elevation ( $\mathrm{n} \%)$ & $112(\% 24.7)$ & $35(\% 29.3)$ & 0.170 \\
\hline $\mathrm{SpO}_{2}(\%)$ & $93(90-94)$ & $93(91-94)$ & 0.167 \\
\hline Respiratory rate ( $\mathrm{n} /$ minute) & $27(26-30)$ & $27(26-29)$ & 0.681 \\
\hline ICU (n \%) & $36(\% 7.9)$ & $9(\% 8)$ & 0.990 \\
\hline Mortality (n \%) & $27(\% 5.9)$ & $7(\% 6.8)$ & 0.920 \\
\hline
\end{tabular}

\section{TABLE 1: The main clinical and biochemical characteristics of the patients received and not}

received favipiravir

Values are presented using means \pm standard deviations for normally distributed and medians and first and third quartiles in the brackets for the nonnormally distributed variables; ALT: alanine aminotransferase; AST: aspartate aminotransferase; CRP: c-reactive protein; WBC: white blood cell; ICU: intensive care unit, $\mathrm{SpO}_{2}$ : peripheral capillary oxygen saturation.

\begin{tabular}{|c|c|c|c|c|c|c|}
\hline & $\begin{array}{l}\text { AST elevated n:165 } \\
(\% 29.1)\end{array}$ & $\begin{array}{l}\text { AST normal n:402 } \\
(\% 70.9)\end{array}$ & $\begin{array}{l}\text { P- } \\
\text { value }\end{array}$ & $\begin{array}{l}\text { ALT elevated n:147 } \\
(\% 25.9)\end{array}$ & $\begin{array}{l}\text { ALT normal n:420 } \\
(\% 74.1)\end{array}$ & $\begin{array}{l}P \text { - } \\
\text { value }\end{array}$ \\
\hline ICU (n \%) & $24(\% 14.5)$ & $21(\% 5.2)$ & 0.001 & $6(\% 4.1)$ & $39(\% 9.3)$ & 0.06 \\
\hline $\begin{array}{l}\text { Mortality (n } \\
\%)\end{array}$ & $22(\% 13.3)$ & $12(\% 3)$ & 0.001 & $5(\% 3.4)$ & $29(\% 6.9)$ & 0.12 \\
\hline
\end{tabular}

TABLE 2: Mortality and ICU admission in patient groups with and without serum transaminase elevation

ALT: alanine aminotransferase; AST: aspartate aminotransferase; ICU: intensive care unit. 


\section{Cureus}

Intensive care unit (\%)

Mortality (\%)

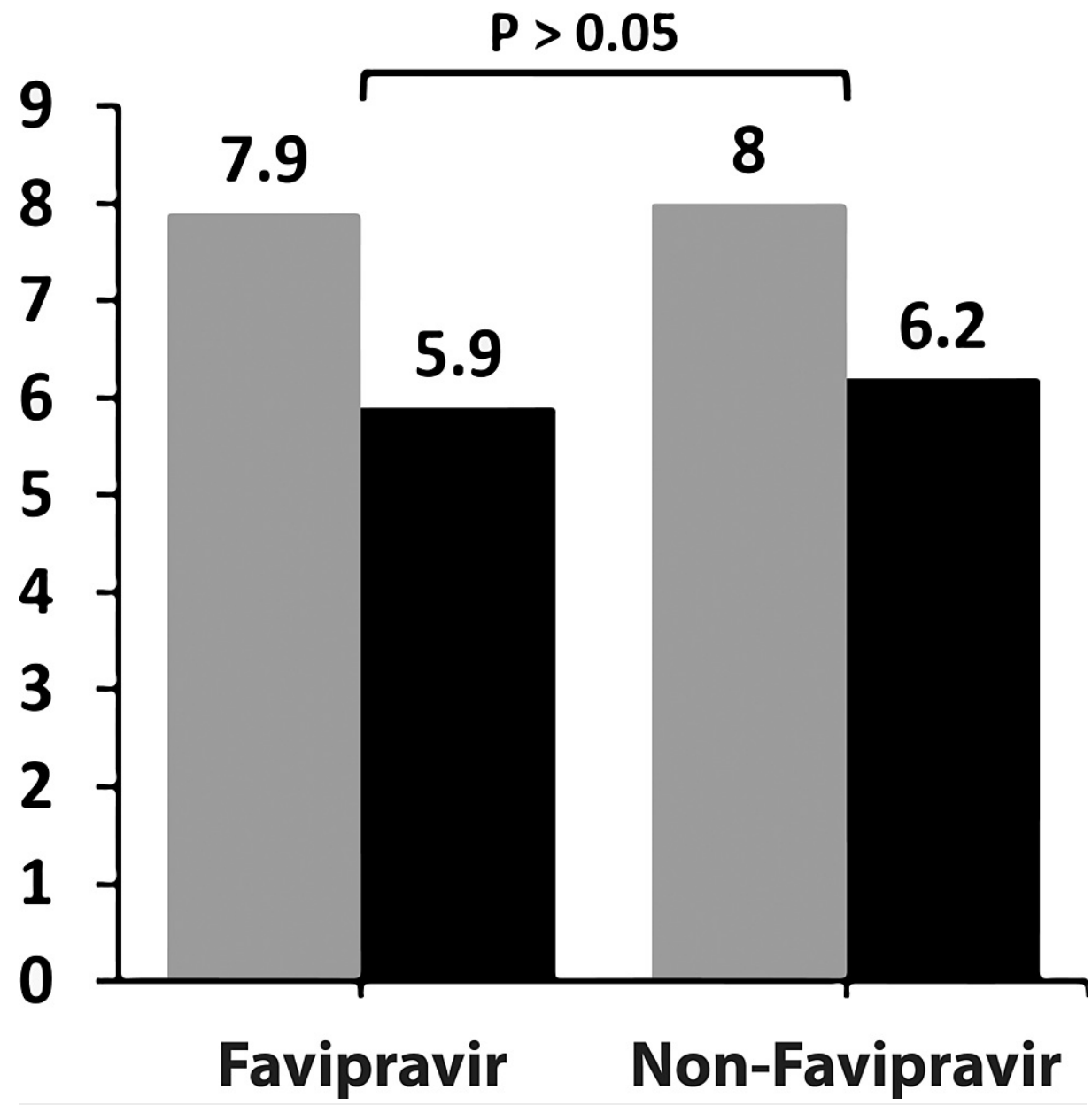

FIGURE 2: Mortality and ICU admission rates in receiving and not receiving favipiravir groups

There was no statistically significant difference between patients who are treated with or without favipiravir in terms of mortality and ICU rates $(p>0.05)$. 


\section{Cureus}

\begin{tabular}{|c|c|c|c|c|c|c|c|c|}
\hline & \multicolumn{4}{|c|}{ Univariate Analysis } & \multicolumn{4}{|c|}{ Multivariate Analysis } \\
\hline & \multirow[b]{2}{*}{ OR } & \multicolumn{2}{|c|}{$\% 95$ confidence interval } & \multirow[b]{2}{*}{$\mathrm{p}$-values } & \multirow[b]{2}{*}{ OR } & \multicolumn{2}{|c|}{$\% 95$ confidence interval } & \multirow[b]{2}{*}{ p-values } \\
\hline & & Lower bound & Upper bound & & & Lower bound & Upper bound & \\
\hline Age & 1.068 & 1.037 & 1.100 & 0.001 & 1.068 & 1.037 & 1.100 & 0.001 \\
\hline CRP & 1.006 & 1.002 & 1.010 & 0.001 & 1.008 & 1.003 & 1.012 & 0.001 \\
\hline Platelet & 0.994 & 0.989 & 0.999 & 0.016 & 0.994 & 0.989 & 0.999 & 0.014 \\
\hline AST & 1.059 & 1.015 & 1.102 & 0.008 & 1.051 & 1.006 & 1.097 & 0.026 \\
\hline
\end{tabular}

TABLE 3: Predictors for mortality in hospitalized patients with COVID infection

AST: aspartate aminotransferase; CRP: c-reactive protein.

\begin{tabular}{|c|c|c|c|c|c|c|c|c|}
\hline & \multicolumn{4}{|c|}{ Univariate analysis } & \multicolumn{4}{|c|}{ Multivariate analysis } \\
\hline & \multirow[b]{2}{*}{ OR } & \multicolumn{2}{|c|}{ \%95 Confidence Interval } & \multirow[b]{2}{*}{$p$ values } & \multirow[b]{2}{*}{ OR } & \multicolumn{2}{|c|}{ \%95 Confidence Interval } & \multirow[b]{2}{*}{$p$ values } \\
\hline & & Lower bound & Upper bound & & & Lower bound & Upper bound & \\
\hline Age & 1.056 & 1.031 & 1.083 & 0,001 & 1.057 & 1.033 & 1.083 & 0.001 \\
\hline CRP & 1.006 & 1.002 & 1.009 & 0.011 & 1.006 & 1.002 & 1.010 & 0.002 \\
\hline Platelet & 0.995 & 0.991 & 0.999 & 0.012 & 0.995 & 0.990 & 0.999 & 0.009 \\
\hline AST & 1.013 & 1.003 & 1.024 & 0.011 & 1.011 & 1.000 & 1.023 & 0.058 \\
\hline
\end{tabular}

TABLE 4: Predictors for ICU admission in hospitalized patients with COVID infection

AST: aspartate aminotransferase; CRP: c-reactive protein.

\section{Discussion}

When the literature is reviewed, this study is the first one that gives priority to the evaluation of the relationship between the use of favipiravir and elevated serum transaminases in the treatment of COVID-19 patients. In our study, no significant difference was found in the development of serum transaminase elevation between COVID-19 patients used and did not use favipiravir. In addition, in our study, AST elevation was found to be a significant risk factor for mortality and ICU admission in COVID-19 patients.

Favipiravir is a prodrug that converts to the active form by intracellular phosphorylation [12]. Metabolized by aldehyde oxidase and partially xanthine oxidase in the liver, it turns into an inactive metabolite and is excreted by the renal route [16]. Metabolism of a drug from the liver affects its hepatotoxicity potential [17]. Serum transaminase level elevation in favipiravir use has been reported as $8.6 \%$ in a clinical trial and as $2 \%$ in a systematic review $[13,14]$. In two different studies, the prevalence of COVID 19-induced elevation of transaminases was found to be $21-46 \%$ for AST and 19-35\% for ALT [2,18]. Similar to these results, in our study, COVID-related transaminase elevation was observed in 25\% and $27 \%$ for AST and ALT, respectively. In addition, this elevation was less than five times the upper limit of normal in all of our patients and none of the patients developed severe transaminase elevation and liver failure. These results were also compatible with the literature data [18]. Current data show that COVID-19 patients with severe clinical findings develop irregular cytokine release, also known as "cytokine storm." Cytokine storm causes an extreme inflammatory and immune response that leads to acute respiratory distress syndrome (ARDS), pulmonary edema, and multi-organ failure. That means the overactive and untreated immune response in COVID-19 can be mortal [19]. Serum transaminase elevation in COVID-19 is associated with the severity of the disease and increased inflammatory markers [18]. Hepatocellular injury can be associated with the direct cytopathic effect of the virus, hyperinflammatory state, thrombotic microangiopathy, and hypoxia secondary to pneumonia $[4,5,18]$. Increased viral load in SARS-CoV-2 is associated with increased IL-6 level, cytokine storm, and poor prognosis [20,21]. Favipiravir is a potent inhibitor of viral proliferation. It shortens the time of viral clearance, possibly decreases cytokine production, and protects the lung from inflammatory damage $[16,22,23]$. Although it is known that favipiravir use causes elevated transaminase levels, no significant 
difference was observed in the elevation of transaminase levels between the patient groups using and not using the drug.

Serum transaminase elevations are indicators of hepatocellular damage and are detected in $8 \%$ of the population [24]. While ALT elevation is observed in clinical pictures such as hepatitis and drug-induced toxicity, AST elevation may also develop secondary to non-hepatic causes such as alcohol-induced hepatitis, hemolysis, myopathy, and exercise [24]. Aminotransferase elevation may be caused by myositis, and in COVID-19, the elevation of creatine kinase was detected in patients at a rate of $14 \%$ [2]. When considering along with the literature data, in our study, AST elevation in COVID-19 patients was evaluated as secondary to disease-related inflammatory response, while ALT elevation was evaluated as secondary to drug-related hepatotoxicity [25]. In our study, a significant relationship was found between AST elevation with mortality and ICU admission. Conversely, the mortality and ICU admission rates did not change in patients with ALT elevation. This contrast between the results also supports the idea mentioned above. CRP and IL-6 levels were detected at the same levels in patients with COVID-19 pneumonia and non-COVID pneumonia [26]. However, higher serum transaminase levels were detected in patients with grade III COVID-19 pneumonia than non-COVID pneumonia patients [27]. In our study, we detected elevated AST levels as a risk factor for mortality due to COVID-19 disease. On the other hand, for serum ALT level, a borderline inverse correlation was detected for the ICU admission, but there was no correlation with mortality. Both the lack of significant elevation in serum transaminases due to favipiravir use and the strong relation of AST value with ICU and mortality compared to ALT suggests that the elevation of serum transaminases in COVID-19 may be primarily due to the hyperinflammatory condition and cytopathic effect of the virus. There is a need for multicenter studies with larger patient numbers on the elevation of transaminases in favipiravir treatment in COVID-19.

The limitations of the study are its retrospective nature and the low number of patients who did not receive favipiravir. However, the fact that the group that did not receive favipiravir had better inflammatory and mortality-related markers such as CRP and lymphocyte compared to the other group may be considered as a limitation. This may be the result of the use of favipiravir in the treatment of more severe COVID-19 patients due to difficulties in drug supply in the early stages of the epidemic.

\section{Conclusions}

In conclusion, there was no statistically significant elevation in serum transaminase levels due to favipiravir use in patients who were hospitalized for COVID-19. According to our study, the use of Favipiravir in the treatment of COVID-19 seems safe in terms of the development of serum transaminase elevation in patients. Additionally, the elevation of AST levels seems to be a significant risk factor for mortality and ICU admission in patients with COVID-19. Patients with elevated AST levels during hospitalization should be followed up more closely by clinicians in terms of the prognosis of the disease. Further multicenter studies with larger patient samples are needed on this subject.

\section{Additional Information \\ Disclosures}

Human subjects: Consent was obtained or waived by all participants in this study. Local Ethical Committee of Kanuni Sultan Süleyman Training and Research Hospital issued approval KAEK/2021.01.18. Animal subjects: All authors have confirmed that this study did not involve animal subjects or tissue. Conflicts of interest: In compliance with the ICMJE uniform disclosure form, all authors declare the following: Payment/services info: All authors have declared that no financial support was received from any organization for the submitted work. Financial relationships: All authors have declared that they have no financial relationships at present or within the previous three years with any organizations that might have an interest in the submitted work. Other relationships: All authors have declared that there are no other relationships or activities that could appear to have influenced the submitted work.

\section{Acknowledgements}

Ali Riza Koksal was supported by funds received from the Akdamar Fellowship Program, Department of Gastroenterology and Hepatology, Tulane University Health Sciences Center.

\section{References}

1. Weiss SR, Leibowitz JL: Coronavirus pathogenesis. Adv Virus Res. 2011, 81:85-164. 10.1016/B978-0-12385885-6.00009-2

2. Guan WJ, Ni ZY, Hu Y, et al.: Clinical characteristics of Coronavirus disease 2019 in China . N Engl J Med. 2020, 382:1708-20. 10.1056/NEJMoa2002032

3. Huang C, Wang Y, Li X, et al.: Clinical features of patients infected with 2019 novel coronavirus in Wuhan, China. Lancet. 2020, 395:497-506. 10.1016/S0140-6736(20)30183-5

4. Zhang C, Shi L, Wang FS: Liver injury in COVID-19: management and challenges . Lancet Gastroenterol Hepatol. 2020, 5:428-30. 10.1016/S2468-1253(20)30057-1

5. Jothimani D, Venugopal R, Abedin MF, Kaliamoorthy I, Rela M: COVID-19 and the liver. J Hepatol. 2020, 73:1231-40. 10.1016/j.jhep.2020.06.006 
6. Fan Z, Chen L, Li J, et al.: Clinical features of COVID-19-related liver functional abnormality . Clin Gastroenterol Hepatol. 2020, 18:1561-6. 10.1016/j.cgh.2020.04.002

7. Abdulla S, Hussain A, Azim D, et al.: COVID-19-induced hepatic injury: a systematic review and metaanalysis. Cureus. 2020, 12:e10923. 10.7759/cureus.10923

8. Hundt MA, Deng Y, Ciarleglio MM, Nathanson MH, Lim JK: Abnormal liver tests in COVID-19: a retrospective observational cohort study of 1,827 patients in a major U.S. hospital network. Hepatology. 2020, 72:1169-76. 10.1002/hep.31487

9. Agrawal U, Raju R, Udwadia ZF: Favipiravir: a new and emerging antiviral option in COVID-19. Med J Armed Forces India. 2020, 76:370-6. 10.1016/j.mjafi.2020.08.004

10. Furuta Y, Gowen BB, Takahashi K, Shiraki K, Smee DF, Barnard DL: Favipiravir (T-705), a novel viral RNA polymerase inhibitor. Antiviral Res. 2013, 100:446-54. 10.1016/j.antiviral.2013.09.015

11. Cai Q, Yang M, Liu D, et al.: Experimental treatment with favipiravir for COVID-19: an open-label control Study. Engineering (Beijing). 2020, 6:1192-8. 10.1016/j.eng.2020.03.007

12. Joshi S, Parkar J, Ansari A, et al.: Role of favipiravir in the treatment of COVID-19 . Int J Infect Dis. 2021, 102:501-8. 10.1016/j.ijid.2020.10.069

13. Pilkington V, Pepperrell T, Hill A: A review of the safety of favipiravir - a potential treatment in the COVID19 pandemic?. J Virus Erad. 2020, 6:45-51. 10.1016/S2055-6640(20)30016-9

14. Chen C, Zhang Y, Huang J, et al.: Favipiravir versus Arbidol for COVID- 19: a randomized clinical trial [PREPRINT]. medRxiv. 2020, 10.1101/2020.03.17.20037432

15. Varghese GM, John R, Manesh A, Karthik R, Abraham OC: Clinical management of COVID-19. Indian J Med Res. 2020, 151:401-10. 10.4103/ijmr.IJMR_957_20

16. Du YX, Chen XP: Favipiravir: pharmacokinetics and concerns about clinical trials for 2019-nCoV infection . Clin Pharmacol Ther. 2020, 108:242-7. 10.1002/cpt.1844

17. European Association for the Study of the Liver: EASL Clinical Practice Guidelines: drug-induced liver injury. J Hepatol. 2019, 70:1222-61. 10.1016/j.jhep.2019.02.014

18. Bertolini A, van de Peppel IP, Bodewes FA, et al.: Abnormal liver function tests in patients with COVID-19: relevance and potential pathogenesis. Hepatology. 2020, 72:1864-72. 10.1002/hep.31480

19. de la Rica R, Borges M, Gonzalez-Freire M: COVID-19: in the eye of the cytokine storm . Front Immunol. 2020, 11:558898. 10.3389/fimmu.2020.558898

20. Chen X, Zhao B, Qu Y, et al.: Detectable serum severe acute respiratory syndrome Coronavirus 2 viral load (RNAemia) is closely correlated with drastically elevated interleukin 6 level in critically ill patients with Coronavirus disease 2019. Clin Infect Dis. 2020, 71:1937-42. 10.1093/cid/ciaa449

21. Ruan Q, Yang K, Wang W, Jiang L, Song J: Clinical predictors of mortality due to COVID-19 based on an analysis of data of 150 patients from Wuhan, China. Intensive Care Med. 2020, 46:846-8. 10.1007/s00134020-05991-X

22. Takahashi H, Iwasaki Y, Watanabe T, et al.: Case studies of SARS-CoV-2 treated with favipiravir among patients in critical or severe condition. Int J Infect Dis. 2020, 100:283-5. 10.1016/j.ijid.2020.08.047

23. Yamamura H, Matsuura H, Nakagawa J, Fukuoka H, Domi H, Chujoh S: Effect of favipiravir and an antiinflammatory strategy for COVID-19. Crit Care. 2020, 24:413. 10.1186/s13054-020-03137-5

24. Lala V, Goyal A, Bansal P, Minter DA: Liver Function Tests. StatPearls [Internet], Treasure Island; 2021.

25. Lei F, Liu YM, Zhou F, et al.: Longitudinal association between markers of liver injury and mortality in COVID-19 in china. Hepatology. 2020, 72:389-98. 10.1002/hep.31301

26. Li Y, Li H, Han J, Yang L: The preliminary comparative results between Covid-19 and non-Covid-19 patients in Western China. BMC Infect Dis. 2020, 20:935. 10.1186/s12879-020-05680-6

27. Zheng Y, Huang Z, Yin G, et al.: Study of the lymphocyte change between COVID-19 and non-COVID-19 pneumonia cases suggesting other besides uncontrolled inflammation contributed to multi-organ injury [PREPRINT]. medRxiv. 2020, 10.1101/2020.02.19.20024885 